\section{Die Tuberkulosesituation in Deutschland 1998}

\section{Einleitung}

Die Tuberkulose ist unverändert weltweit bei Jugendlichen und Erwachsenen die am häufigsten zum Tode führende Infektionskrankheit. Ein Viertel aller vermeidbaren Todesfälle auf der Welt wird durch Tuberkuloseinfektionen verursacht. Jährlich sterben 2-3 Millionen Menschen, darunter 100000 Kinder, an dieser Erkrankung. Die Weltgesundheitsorganisation (WHO) schätzt, dass aktuell nahezu ein Drittel der Weltbevölkerung infiziert ist. Von den 100 Millionen Menschen, die sich jährlich neu mit Mycobacterium tuberculosis infizieren, erkranken im Laufe ihres Lebens ca. 5-10\% an einer aktiven Tuberkulose. Die Zahl der jährlichen Neuerkrankungen wird weltweit auf insgesamt 7-8 Millionen geschätzt. Über 95\% aller Tuberkulosefälle treten in Entwicklungsländern auf. Wie in den Vorjahren steigen die Tuberkuloseinzidenzen in Osteuropa und der ehemaligen Sowjetunion seit dem Zusammenbruch der politischen Systeme und damit der medizinischen Versorgungsstrukturen dramatisch an.

Begünstigt durch Faktoren wie HIV-Infektion, zunehmende Verarmung, Migration, medizinische Unterversorgung und unzureichende Umsetzung internationaler Empfehlungen im Rahmen nationaler Tuberkuloseprogramme ist die Tuberkulose weltweit weiter auf dem Vormarsch. Etwa ein Drittel der Zunahme der Erkrankungshäufigkeit in den letzten fünf Jahren wird von der WHO auf die Ko-Morbidität mit HIV zurückgeführt.

Von besonderer Bedeutung für die Erkrankungssituation auch in Deutschland - ist die dramatische Zunahme der Tuberkuloseerkrankungen und -mortalität in Osteuropa, und hier insbesondere in den GUS-Staaten. Aus Osteuropa wird gegenwärtig eine Viertelmillion neuer Tuberkulosefälle pro Jahr berichtet. Die Inzidenzraten in Osteuropa liegen durchschnittlich bei 43,3 pro 100000 Einwohner [9]. Inadäquate Therapie durch fehlerhafte Verordnungen, Mangel an Medikamenten, ungenügende Therapiekontrolle und fehlende Compliance der Patienten haben zudem dazu geführt, dass medikamentenresistente Tuberkulosen insbesondere in Osteuropa zugenommen haben. Schätzungen gehen von weltweit 50 Millionen Menschen aus, die mit resistenten Bakterienstämmen infiziert sind.

Pneumologie 2000; 54: 324-332

(c) Georg Thieme Verlag Stuttgart · New York ISSN 0934-8387
R. Loddenkemper, D. Sagebiel, C. Meyer, B. Hauer, M. Forßbohm ${ }^{1}$

Deutsches Zentralkomitee zur Bekämpfung der Tuberkulose

(DZK), Lungenklinik Heckeshorn, Berlin

${ }^{1}$ Gesundheitsamt Wiesbaden

Die seit 1996 durchgeführte DZK-Studie (Studienleiter: Dr. M. Forßbohm) zur Epidemiologie der Tuberkulose in Deutschland, an der $2 / 3$ aller deutschen Gesundheitsämter teilnehmen und die $60 \%$ aller Patienten in Deutschland erfasst, beschreibt für 1998 Resistenzraten von 31,5\% gegen mindestens ein Tuberkulose-Erstrangmedikament für in den GUS-Staaten geborene Tuberkulosepatienten, die in Deutschland leben. Die Multiresistenz (MDR), d.h. Resistenz gegen mindestens INH und RMP, stieg in dieser Gruppe 1998 auf 11,8\% an, während die MDR in Deutschland insgesamt von 1,2\% (1996) auf 1,4\% (1998) anstieg. Die Resistenzsituation in der deutschen Bevölkerung stellt sich in dieser Studie vergleichsweise stabil dar [1].

Die epidemiologische Situation der Tuberkuloseerkrankungen in Deutschland ist seit Jahren stabil. Die Gesamtinzidenz für 1998 beträgt 12,7 pro 100000 Einwohner gegenüber 13,6 pro 100000 Einwohner im Jahr 1997. Dieser insgesamt rückläufige Trend muss jedoch das Augenmerk vermehrt auf Risikogruppen mit erhöhter Auftretenswahrscheinlichkeit für Tuberkuloseerkrankungen in Deutschland lenken. Neben der Kontrolle und effizienten Bekämpfung muss das Erreichte durch gezielte präventive und diagnostische Maßnahmen stabilisiert werden sowie insbesondere mit neuen gesundheitspolitischen Handlungsanweisungen den akuten epidemiologischen Tendenzen und Erfordernissen angepasst werden. Effektive, kostengünstige und erfolgreiche Prävention und Therapie von Tuberkulose erfordert deshalb auch eine fundierte Datengrundlage zur Untermauerung neuer Handlungsanweisungen. Hierfür wird das neue Infektionsschutzgesetz eine wichtige Grundlage schaffen.

Im folgenden sollen die Daten des 25. Informationsberichtes des DZK für das Jahr 1998 unter besonderer Berücksichtigung der bekannten Risikopopulationen dargestellt werden. Die Zahlenangaben aus der Studie wurden aktualisiert.

\section{Die Tuberkulosesituation in der Bundesrepublik Deutschland 1998}

1998 wurden in der Bundesrepublik Deutschland 10440 neue Erkrankungsfälle registriert. Dies entspricht bei einer Gesamtbevölkerung von 82037011 Menschen einer Inzidenz von 12,7 pro 100000 Einwohner. Gegenüber 1997 traten 723 Fälle weniger auf $(-6,5 \%)$. Den Verlauf der Tuberkuloseneuerkrankungen für die einheimische und ausländische Bevölkerung seit 1974 zeigt Abb. 1. Nur in den Jahren 1992 und 1993 war ein geringer Anstieg der Gesamtinzidenz zu verzeichnen. Der Hauptgrund für diesen Anstieg war die Zunahme der Tuber- 

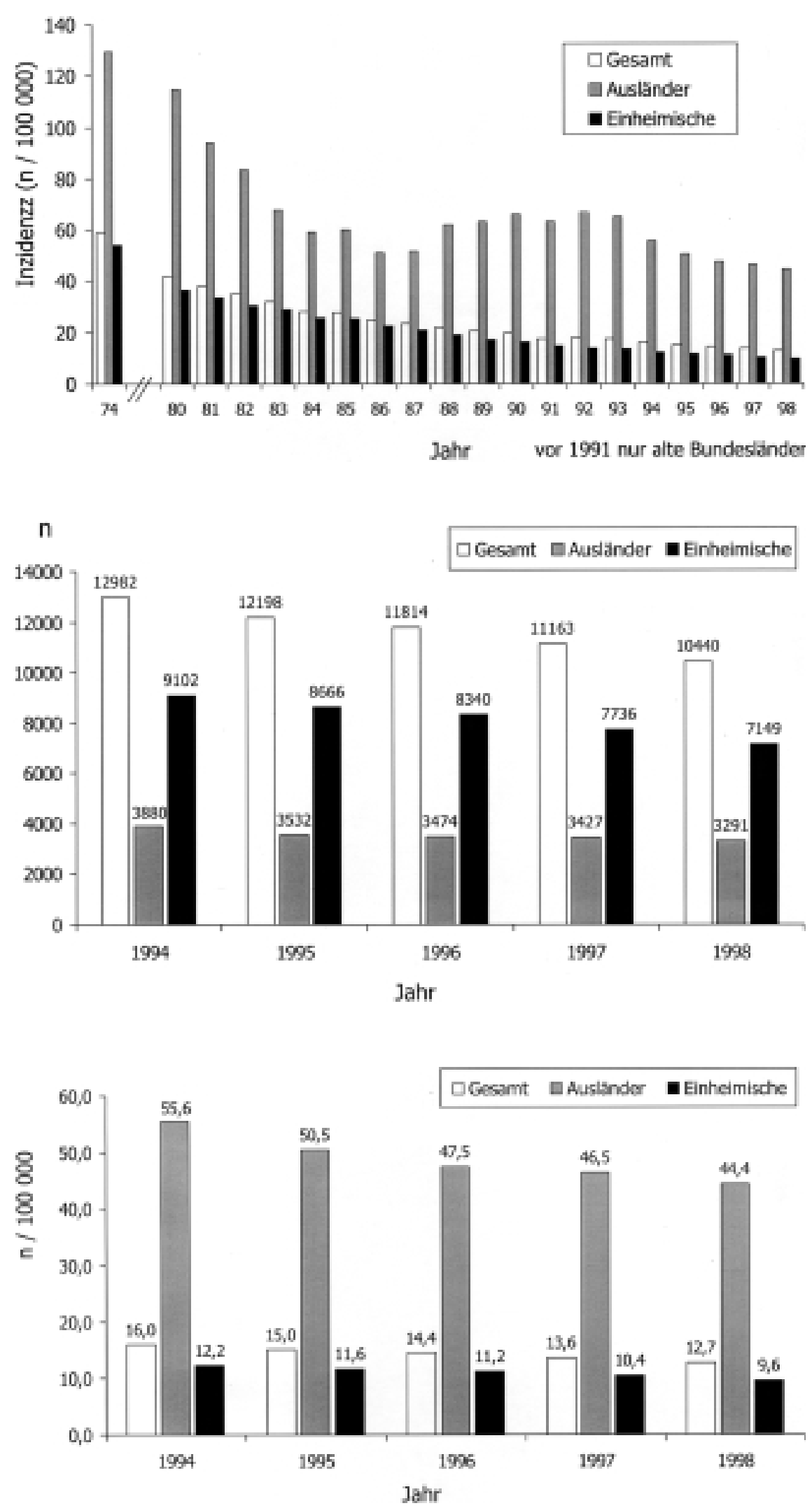

Abb. 1 Entwicklung der Tuberkuloseinzidenz in Deutschland 1974-1998
Abb. 2 Neuerkrankungen an Tuberkulose (Absolutzahlen) (1994-1998)

Abb. 3 Neuerkrankungen an Tuberkulose (Inzidenz) (1994-1998)

kuloseerkrankungen in der ausländischen Bevölkerung, da in diesem Zeitraum der Zustrom von Migranten besonders ausgeprägt war. Den Vergleich der absolut Erkrankten und der
Inzidenzen für die Gesamtbevölkerung sowie die einheimische und ausländische Bevölkerung von 1994 bis 1998 stellen Abb. 2 und 3 dar. 


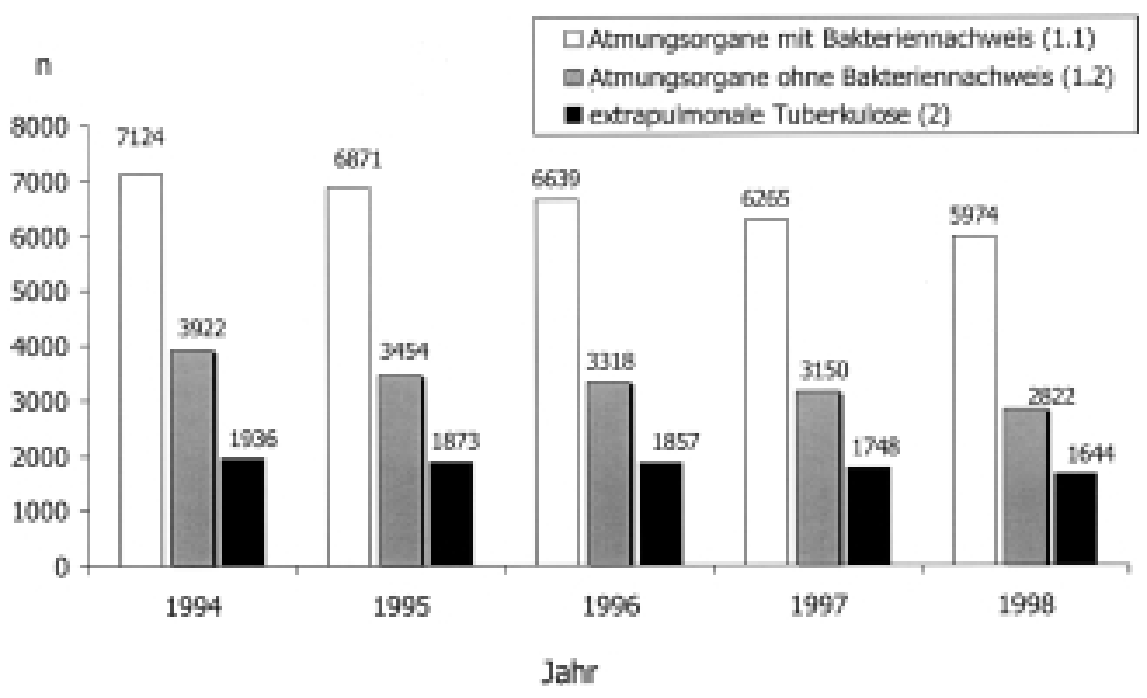

Abb. 4 Neuerkrankungen an Tuberkulose (Absolutzahlen) nach Diagnosegruppen

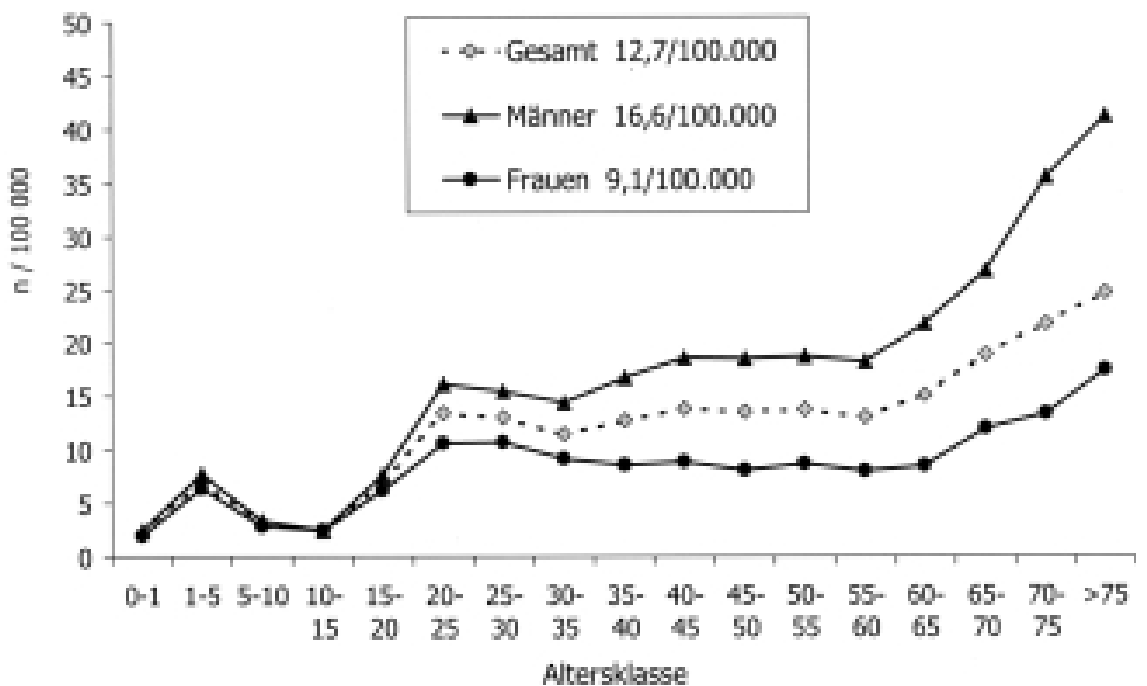

Abb. 5 Tuberkuloseinzidenzen (alle Formen) in Deutschland 1998 nach Geschlecht und Alter

Im Vergleich zu den Vorjahren hat sich auch die Verteilung auf die einzelnen Tuberkuloseformen nicht wesentlich verändert: Auf eine Tuberkulose der Atmungsorgane mit Nachweis von Tuberkulosebakterien (Diagnosegruppe 1.1) entfielen 5974 Fälle (57,2\%), auf eine Tuberkulose der Atmungsorgane ohne Nachweis von Tuberkulosebakterien (Diagnosegruppe 1.2) 2822 Fälle (27\%). Auf eine Tuberkulose anderer Organe (Diagnosegruppe 2) entfielen dagegen 1644 Fälle (15,7\%) (vgl. auch Abb. 4). Unter den 5974 offen-tuberkulösen Fällen gelang bei 3124 (52,3\%) der direkte Nachweis von Tuberkulosebakterien im Sputum. Dies ist der epidemiologisch entscheidende, da besonders ansteckungsfähige, Anteil an allen Tuberkuloseformen.

Der weiterhin leichte Rückgang an Neuerkrankungen ist 1998 vor allem durch die Abnahme geschlossener Tuberkuloseformen bedingt. Die Zahl der offenen Tuberkuloseformen ist im Vergleich zu 1997 um 4,6\%, die der geschlossenen Tuberkuloseformen um 10,4\% und die der extrapulmonalen Tuberkulosen um $5,9 \%$ zurückgegangen.
Wie auch in den Vorjahren erkrankten Männer doppelt so häufig an Lungentuberkulose wie Frauen (67,2\% vs. 32,8\%), die Inzidenz der Frauen betrug 9,1/100 000 Einwohner, die der Männer 16,6/100000 (vgl. Abb.5). Für die extrapulmonalen Tuberkuloseformen gilt, dass Frauen auch hier weiterhin etwas häufiger erkrankten (56,4\%). Die Verteilung innerhalb der extrapulmonalen Formen stellt sich im wesentlichen unverändert dar. Am häufigsten wurde die periphere Lymphknotentuberkulose mit 46,9\% beobachtet, gefolgt von der Urogenitaltuberkulose mit 22,9\%, und in $12,3 \%$ waren Knochen und Gelenke betroffen. Eine Meningitis tuberculosa fand sich in 4,1\% der Fälle (68 Personen, darunter ein einheimisches und vier ausländische Kinder), und 13,7\% der extrapulmonalen Tuberkulosen entfielen auf sonstige Organe (Abb. 6).

\section{Die Altersverteilung der Tuberkuloseerkrankungen}

An Tuberkulose erkrankten unverändert am häufigsten ältere Männer. Männer, die älter als 75 Jahre waren, zeigten die höchste Inzidenz, an Tuberkulose zu erkranken (41,2/100 000, Abb. 5). Die Zusammenhänge von Inzidenz und Mortalität in 

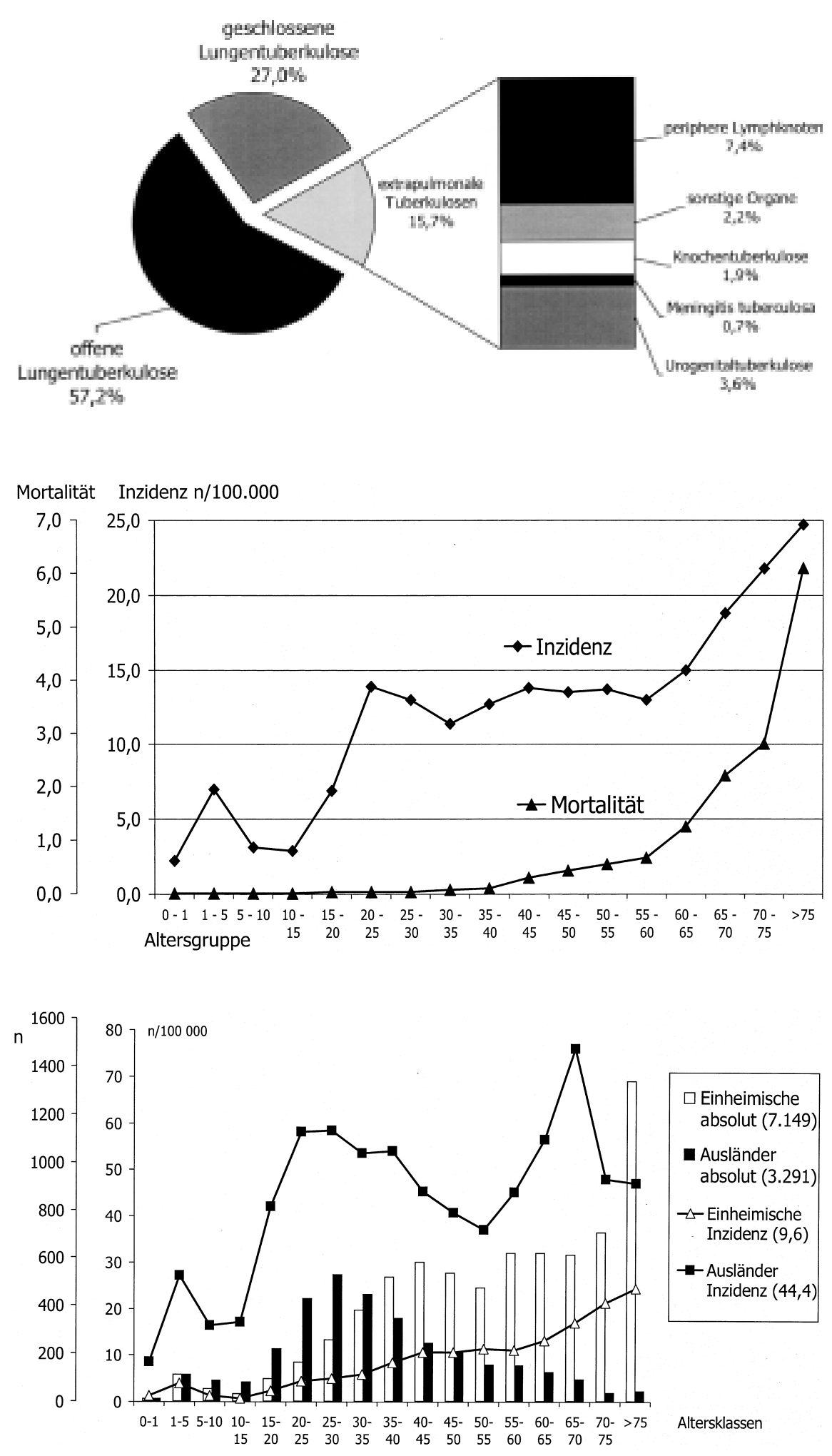

Abb. 6 Verteilung der Tuberkuloseformen $1998(n=10440)$

Abb. 7 Neuerkrankung und Sterblichkeit für alle Tuberkuloseformen 1998

Abb. 8 Tuberkuloseinzidenzen und Absolutzahlen 1998 in Deutschland nach Altersgruppen für Ausländer und Einheimische
Abhängigkeit von den einzelnen Altersgruppen werden in Abb. 7 gezeigt.

Abb. 8 zeigt die Altersverteilung für die einheimische und die ausländische Bevölkerung. Bei der einheimischen Bevölkerung nimmt die Inzidenz nahezu kontinuierlich mit höherem Alter zu, während sich bei der ausländischen Bevölke- rung drei Inzidenzgipfel finden: Ausländische Kleinkinder (1-5 Jahre), jüngere Erwachsene (20-40 Jahre) und ausländische Mitbürger über 65 Jahre zeigen im Vergleich zu den anderen Altersgruppen deutlich erhöhte Inzidenzen (die altersabhängigen Inzidenzraten sind in Abb. 8 als unstandardisierte Inzidenzraten für die einzelnen Altersgruppen abzulesen). 


\section{Die Mortalität der Tuberkulose}

1998 starben in der Bundesrepublik Deutschland 711 Menschen an einer Tuberkulose oder deren Spätfolgen. Dies waren 11,7\% weniger als 1997 (805 Sterbefälle). Den „primären Phthisentod" starben 541 Personen, während 170 Personen an den Spätfolgen einer Tuberkulose verstarben. Der Anteil der Sterbefälle an aktiver Tuberkulose blieb mit 5,2\% konstant. Die Mortalität der Tuberkulose betrug damit 0,87 pro 100000 Einwohner und blieb gegenüber den Vorjahren nahezu unverändert (Tab. 1, Abb. 7).

\section{Die Tuberkulosesituation in den einzelnen Bundesländern 1995- 1998}

Tab. 1 Neuerkrankungen und Sterbefälle an Tuberkulose 1993-1998

\begin{tabular}{lllll}
\hline & $\begin{array}{l}\text { Tuberkulose- } \\
\text { erkrankungen }\end{array}$ & \multicolumn{2}{l}{$\begin{array}{l}\text { Sterbefälle an } \\
\text { aktiver Tuberkulose }\end{array}$} & $\begin{array}{l}\text { Sterbefälle } \\
\text { einschließlich } \\
\text { Spätfolgen } \\
\text { n }\end{array}$ \\
\hline 1993 & n & $n$ & $\%$ & 1037 \\
1994 & 14982 & 774 & 5,5 & 1014 \\
1995 & 12198 & 709 & 5,8 & 935 \\
1996 & 11814 & 692 & 5,8 & 896 \\
1997 & 11163 & 593 & 5,3 & 805 \\
1998 & 10440 & 541 & 5,2 & 711 \\
\hline
\end{tabular}

Für das Jahr 1998 ließ sich die für die gesamte Bundesrepublik rückläufige Neuerkrankungsrate auch für die einzelnen Bundesländer nachweisen, lediglich im Saarland nahm die Tuberkuloseinzidenz von 12,7 auf 13,6 pro 100000 Einwohner in geringem Ausmaß zu. Besonders auffallend sind die Rückgänge der Neuerkrankungsraten in den Stadtstaaten Hamburg (Rückgang von 19,2 auf 16,8 pro 100000 Einwohner), Bremen (15,1 auf 13,0 pro 100000) und Berlin (16,1 auf 15,4 pro 100000). Die Bundesländer Hamburg, Hessen, Berlin und Nordrhein-Westfalen lagen allerdings mit ihren Inzidenzraten deutlich über dem Bundesdurchschnitt, während BadenWürttemberg, Thüringen, Brandenburg, Niedersachsen und Schleswig-Holstein deutlich darunter lagen. Dies galt insbesondere auch für Neuerkrankungen an offener Tuberkulose (Bundesdurchschnitt 1998 7,3/100 000) [1]. In Abb.9 sind die Inzidenzen der einzelnen Bundesländer von 1996 bis 1998 vergleichend aufgeführt. Die Karte in Abb.10 zeigt die Kreise der Bundesrepublik mit Inzidenzen > 15,5/10000 Einwohnern (Kreise mit den 20\% höchsten Tuberkuloseinzidenzen).

\section{Die Tuberkulose der in der Bundesrepublik lebenden Ausländer}

In der Bundesrepublik Deutschland lebten am 31.12.1998 insgesamt 7319593 Ausländer (Ausländeranteil an der Gesamtbevölkerung 8,9\%) [2]. Bezüglich epidemiologischer Aussagen ist zu berücksichtigen, dass die Tuberkulosemeldestatistik lediglich die Staatsangehörigkeit - und nicht das Geburtsland - berücksichtigt. Die Gruppe der Spätaussiedler wird somit aufgrund ihrer deutschen Staatsbürgerschaft zur einheimischen Bevölkerung gezählt.

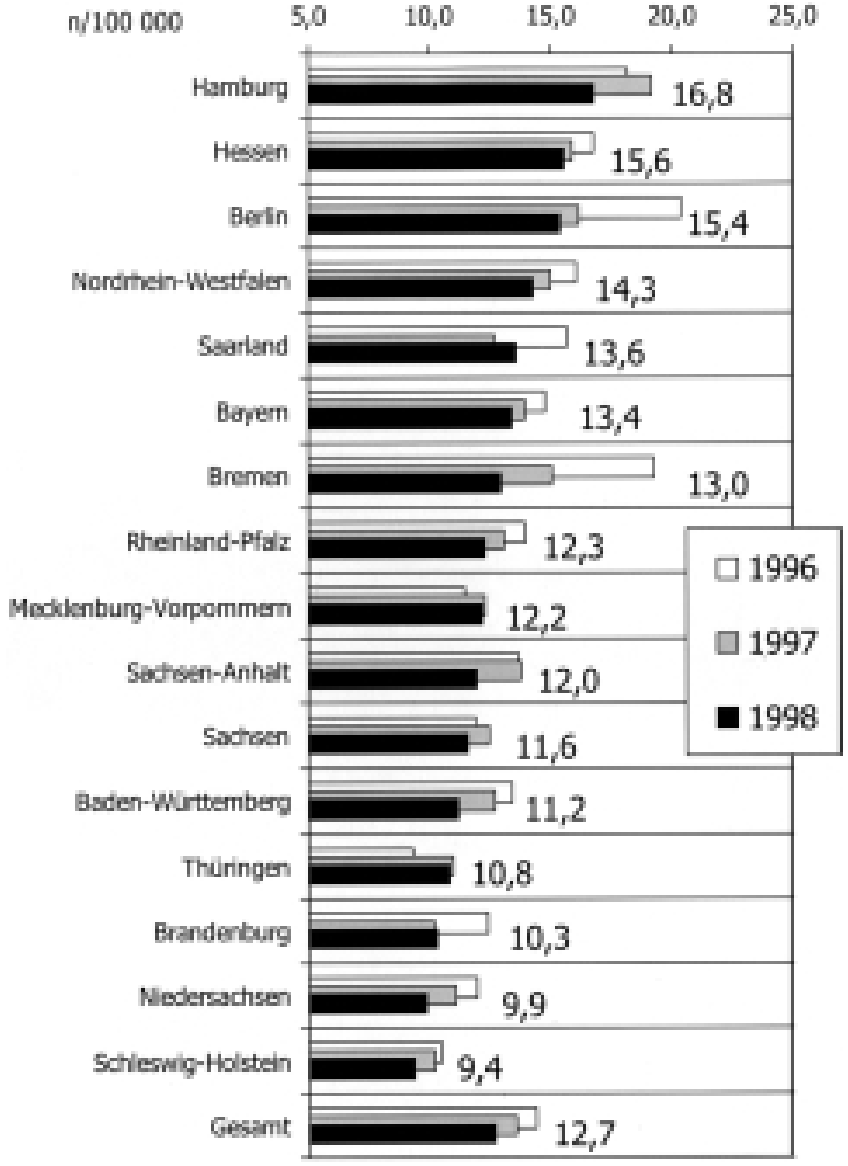

Abb. 9 Tuberkuloseinzidenzen (alle Formen, Gesamtbevölkerung) für die einzelnen Bundesländer 1996-1998

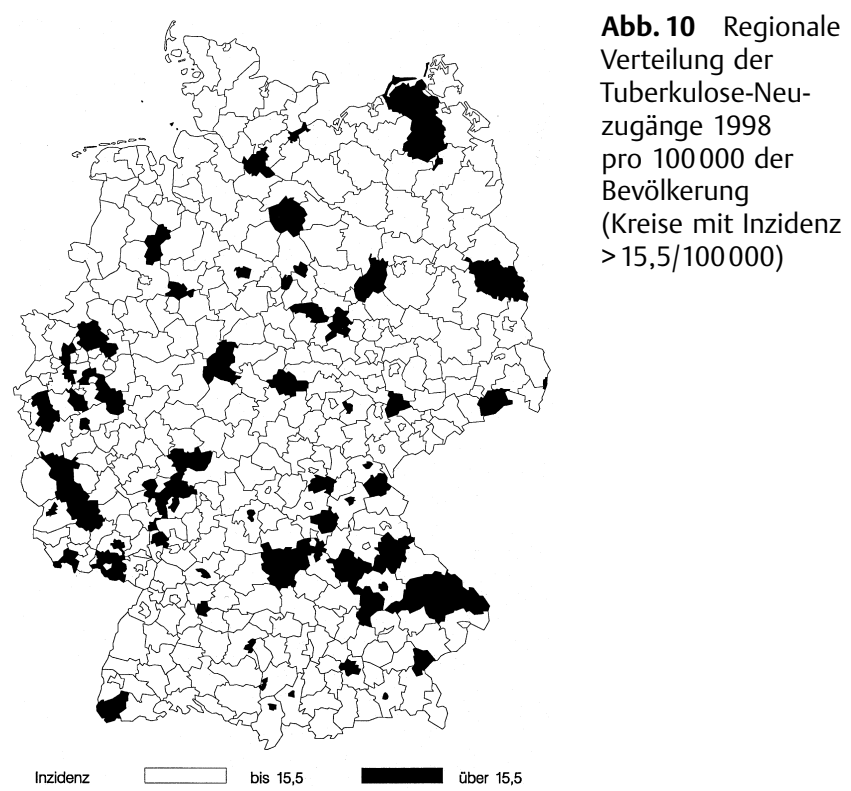

Mehr als 2,1 Millionen Ausländer kamen aus der Türkei, 1,2 Millionen aus dem ehemaligen Jugoslawien (einschließlich Bosnien-Herzegowina, Kroatien, Mazedonien und Slowenien), gefolgt von 612000 Italienern und 363000 Griechen. Insgesamt stammten 81,1\% der in Deutschland lebenden Ausländer 
aus Europa (5983649), 10,9\% aus Asien (796254), 4,1\% aus Afrika (303269) und 3,8\% (281421) aus sonstigen Ländern [3]. 1998 stellten 98644 Personen einen Antrag auf Asyl; der größte Teil der Antragsteller kam aus Osteuropa (41017, insbesondere aus dem ehemaligen Jugoslawien) und den asiatischen Ländern (31971).

Unter den 1998 an Tuberkulose Erkrankten befanden sich 3291 Ausländer (31,5\%), 136 Personen weniger als 1997 ($4,0 \%$ ), was einer Inzidenz von 44,4 pro 100000 Ausländer entsprach. Damit lag die Inzidenz 4,7fach höher als die der einheimischen Bevölkerung (9,6/100000). Auch für die einheimische Bevölkerung war im Vergleich zu 1997 ein Rückgang der Neuerkrankungen zu verzeichnen (587 Fälle weniger als $1997 ;-7,6 \%)$.

Die Inzidenz der Erkrankung an einer offenen Tuberkulose war 1998 für die in der Bundesrepublik lebenden Ausländer mit 22/100000 etwa 3,8fach höher als die der einheimischen Bevölkerung (5,8/100000). 1616 Fälle ließen sich der Diagnosegruppe 1.1 zuordnen (49,1\%), 1010 Fälle der Diagnosegruppe $1.2(30,7 \%)$, und 665 Fälle (20,2\%) fielen unter die Diagnosegruppe 2. Bei den Einheimischen betrug der Anteil offener Tuberkulosen 61,0\%, geschlossener Formen 25,3\% und extrapulmonaler Tuberkulosen $13,7 \%$.

Auch in der ausländischen Bevölkerung erkrankten Männer doppelt so häufig an Lungentuberkulose wie Frauen, während extrapulmonale Tuberkulosen bei Männern und Frauen gleich häufig auftraten.

\section{Die Tuberkulose im Kindesalter}

In der statistischen Erfassung werden Personen zum Kindesalter gerechnet, die das 15. Lebensjahr noch nicht vollendet haben. 1998 lebten insgesamt 13096575 Kinder in Deutschland [2].

In Deutschland erkrankten 1998 insgesamt 490 Kinder an einer aktiven Tuberkulose, dies entspricht einer Inzidenz von 3,74/100000 Kinder (Abb. 11). Im Vergleich zu 1997 wurden somit zusätzliche 10 Fälle gemeldet $(+2,1 \%)$. Nach einem Anstieg der Inzidenz von 1995 (3,87/100000) zu 1996 (4,37/ $100000)$ und einem Rückgang 1997 (3,64/100 000) war 1998 erneut eine leichte Zunahme zu beobachten. Der Anstieg lässt

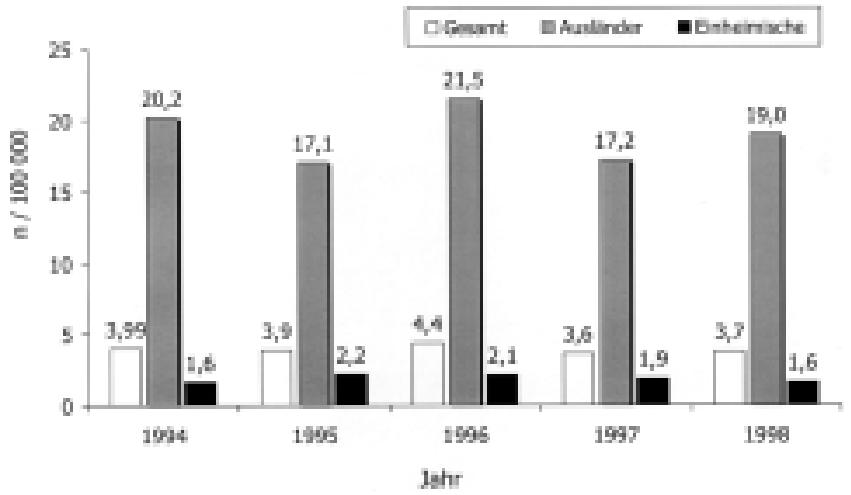

Abb. 11 Tuberkuloseinzidenzen beim Kind in Deutschland: Gesamt, Ausländer, Einheimische (1994-1998) sich vor allem auf eine Zunahme der extrapulmonalen Fälle bei den ausländischen Kindern zurückführen (+ $65,5 \%)$, wogegen bei den einheimischen Kindern insgesamt ein Rückgang um 5,5\% verzeichnet werden konnte. Ausländische Kinder hatten ein 10,5fach höheres Risiko, an Tuberkulose zu erkranken, als einheimische Kinder. Im Vergleich zu 1997 hat die Inzidenz bei Säuglingen von 2,0 auf 2,2/100000 leicht zugenommen (18 Säuglinge, davon 9 ausländische).

Der größte Teil der Erkrankungen im Kindesalter (65,3\%) fand sich auch 1998 unter der Diagnosegruppe 1.2 (Tuberkulose der Atmungsorgane ohne Nachweis von Tuberkulosebakterien, $\mathrm{n}=320$ ); unter die Diagnosegruppe 1.1 (Tuberkulose der Atmungsorgane mit Nachweis von Tuberkulosebakterien, $\mathrm{n}=82$ ) fielen $16,7 \%$ und unter die Diagnosegruppe 2 (extrapulmonale Tuberkulosen) $18 \%$ der Fälle $(n=88)$.

Der Anteil offener Fälle an der Gesamtzahl der Lungentuberkulosen betrug 20,4\% (82/402), davon waren 57 Kinder Ausländer (69,5\%). In nur $24,4 \%$ der Fälle (20/82) gelang dabei der Nachweis von $M$. tuberculosis direkt im Sputumausstrich. Somit fand sich lediglich bei $4,1 \%$ aller an Tuberkulose erkrankten Kinder eine Lungentuberkulose mit positivem mikroskopischen Befund im Sputum (20/490).

Bei den extrapulmonalen Tuberkulosen (88 Fälle) war 1998, wie in den Vorjahren, die Tuberkulose der peripheren Lymphknoten mit 61 Fällen (69,3\%) am häufigsten, darunter waren 27 ausländische Kinder. Es folgte mit 12 Fällen (13,6\%) die Diagnosegruppe „Tuberkulose der sonstigen Organe“, wobei hier die statistische Erfassung keine weitere Differenzierung ermöglicht (z.B. Miliartuberkulose). An einer Knochentuberkulose erkrankten 7 Kinder (8\%). Drei Kinder (3,4\%) erkrankten an einer Urogenitaltuberkulose. Abb.12 zeigt die unterschiedliche Verteilung der Tuberkuloseformen für einheimische und ausländische Kinder. Bei einheimischen Kindern wurden anteilig mehr Tuberkulosen der peripheren Lymphknoten beobachtet.

An einer Meningitis tuberculosa erkrankten 1998 insgesamt 5 Kinder (5,7\% der extrapulmonalen Tuberkulosen), darunter 4 Kinder ausländischer Herkunft. Die Inzidenz ausländischer Kinder mit tuberkulöser Meningitis betrug somit 0,26/ 100000 (1997: 0,13/100000), die einheimischer Kinder 0,008/ 100000 (1997: 0,03/100000). Die Gesamtinzidenzen der letzten Jahre sind stabil.

1998 lebten 1511929 ausländische Kinder in Deutschland (11,5\%). Von den 490 erkrankten Kindern waren 287 Ausländer (58,6\%). Die Inzidenz war im Vergleich zu 1997 bei den ausländischen Kindern mit 19,0/100000 zunehmend, bei den einheimischen Kindern mit 1,8/100000 gering rückläufig (1997: 17,2/100 000 bzw. 1,9/100000) (Abb. 11). Bei den 1-5jährigen ausländischen Kindern war sie mit 27,1/100000 Kinder dieser Altersgruppe am höchsten (1997: 28,8/ 100000); während sie bei einheimischen Kindern dieser Altersgruppe 4,0/100000 betrug. Somit lag das Risiko ausländischer Kinder, an einer Tuberkulose zu erkranken, je nach Altersgruppe um ein 7- bis 20faches höher als das einheimischer Kinder.

Aufgrund konstant niedriger Tuberkuloseinzidenzen und aktueller Nutzen-Risiko-Abwägungen wird von der ständigen 
Einheimische

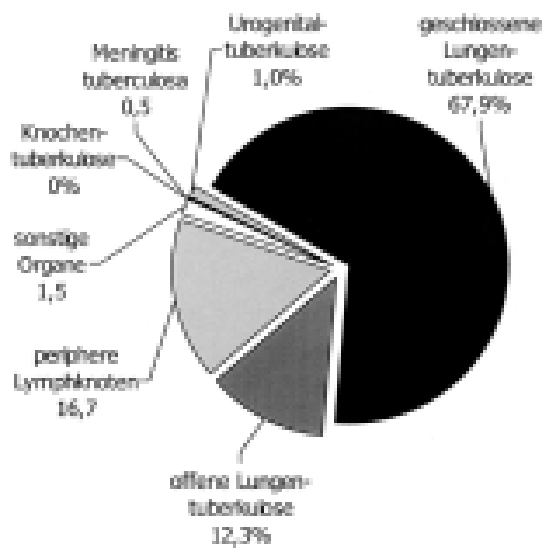

Ausländer

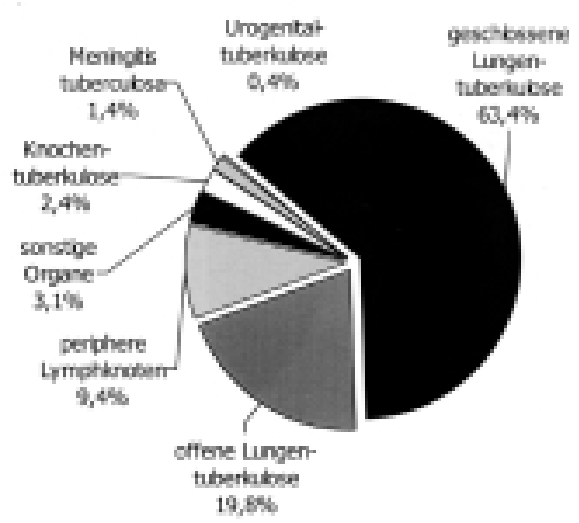

Abb. 12 Vergleich der Tuberkuloseformen bei ausländischen und einheimischen Kindern in Deutschland 1998
Impfkommission am Robert Koch-Institut (STIKO) die BCGImpfung in Deutschland schon seit März 1998 nicht mehr empfohlen [4].

Eine Hauttestung (Mendel-Mantoux) von Kindern aus Risikopopulationen mit engem Kontakt zu Personengruppen mit erhöhter Tuberkuloseinzidenz sollte regelmäßig erfolgen. Für Kinder aus Niedrigrisikogruppen kann eine periodische Hauttestung durchgeführt werden $[11,12]$.

\section{Resistenz von M. tuberculosis-Stämmen in Deutschland}

Im Folgenden sind die Ergebnisse der Umfrage des Arbeitskreises Mykobakterien (AKM) [5] zu Empfindlichkeitsprüfungen von M. tuberculosis-Komplex (Erstisolate) für 1991 - 1998 im Vergleich mit den Daten der DZK-Studie zur Epidemiologie der Tuberkulose $[6,7,8]$ dargestellt (Tab. 2,3).

Die Laboratorien sind jeweils aufgefordert, dem AKM nur den Befund des ersten Stammes, der von einem Kranken isoliert wurde, zu berichten. Die Zahl der Stämme entspricht also ungefähr der Zahl der Patienten. Dabei ist nicht auszuschließen, dass Patienten, deren Isolate in verschiedenen Laboratorien untersucht werden, mehrfach in die Zählung eingehen. Die Resistenzraten für einzelne Antituberkulotika sind als summarische Werte zu verstehen, d.h. sie schließen sowohl Einzel- als auch Mehrfachresistenzen ein. Seit 1995 werden nur die Ergebnisse derjenigen Laboratorien aufgenommen, welche den Qualitätssicherungstest (Ringversuch) bestanden haben.

In den letzten Jahren ist in Deutschland sowohl für Erstrangmedikamente als auch für weitere Medikamente und Medikamentenkombinationen eine kontinuierliche Zunahme der Resistenzen zu beobachten. Resistenzen gegen mindestens ein Erstrangmedikament wurden 1996 in 6,8\%, 1997 in 7,3\% und 1998 in 8,9\% der Resistenztestungen beobachtet.

Auch die Umfrageergebnisse des AKM zeigen im Vergleich zu den Vorjahren eine Zunahme der resistenten Stämme, dieser Trend war 1998 allerdings uneinheitlich. Bei den Mehrfachresistenzen fand sich ein leichter Rückgang, wobei zu berücksichtigen ist, dass es sich um Aussagen über relativ kleine Fallzahlen handelt. Es ist anzunehmen, dass es sich vorwiegend nicht um primäre Resistenzen handelt. Daten zur Vorbehandlung der betroffenen Patienten stehen jedoch nicht zur Verfügung.

Die Daten des Nationalen Referenzzentrums (NRZ) für Mykobakterien in Borstel zeigten 1998 bei 2311 untersuchten Stämmen noch höhere Resistenzraten (H: 11,8\%; R: 5,1\%; E: 3,1\%; Z: 2,1\% und H+R: 4,8\%). Bei vergleichbarem Einsendeklientel wurde in den vergangenen Jahren in Borstel eine kontinuierliche Zunahme der Resistenzdaten registriert. Da in Borstel aber überdurchschnittlich viele Stämme von Patienten eingehen, bei denen eine therapeutische Problematik besteht,

Tab. 2 Resistenzen gegen Antituberkulotika (DKZ-Studie, Stand Juni 2000)

\begin{tabular}{|c|c|c|c|c|c|c|c|c|c|}
\hline Jahr & $\begin{array}{l}\text { Zahl der teilnehmen- } \\
\text { den Labore }\end{array}$ & $\begin{array}{l}\text { Zahl der } \\
\text { Stämme }^{1}\end{array}$ & $\begin{array}{l}\mathrm{H}_{\mathrm{r}} \\
\%\end{array}$ & $\begin{array}{l}\mathrm{R}_{\mathrm{r}} \\
\%\end{array}$ & $\begin{array}{l}\mathrm{S}_{\mathrm{r}} \\
\%\end{array}$ & $\begin{array}{l}\mathrm{E}_{\mathrm{r}} \\
\%\end{array}$ & $\begin{array}{l}Z_{r}^{2} \\
\%\end{array}$ & $\begin{array}{l}\mathrm{H}_{\mathrm{r}}+\mathrm{R}_{\mathrm{r}}\left(+\mathrm{X}_{\mathrm{r}}\right) \\
\%\end{array}$ & $\begin{array}{l}\text { jegliche } \\
\text { Resistenz }^{3}\end{array}$ \\
\hline DZK 1996 & - & 2720 & 5,0 & 1,5 & 3,5 & 1,5 & 1,9 & 1,2 & 6,8 \\
\hline DZK 1997 & - & 3170 & 5,4 & 1,6 & 3,9 & 1,4 & 1,8 & 1,3 & 7,3 \\
\hline DZK 1998 & - & 3271 & 6,2 & 1,7 & 5,1 & 1,5 & 1,8 & 1,5 & 8,9 \\
\hline
\end{tabular}

$\mathrm{H}=\mathrm{INH}, \mathrm{R}=\mathrm{RMP}, \mathrm{S}=\mathrm{SM}, \mathrm{E}=\mathrm{EMB}, \mathrm{Z}=\mathrm{PZA}$

1 M. tuberculosis-Komplex, Resistenz mindestens gegen INH und RMP bekannt

2 M. bovis wurde nicht gezählt

3 Monoresistenz gegen Pyrazinamid betrug 1996: 1,1\%, 1997: 1,3\%, 1998: 1,5\%, wurde bei der Kalkulation der Resistenzrate hier nicht berücksichtigt. 


\section{Allgemeine Resistenz}

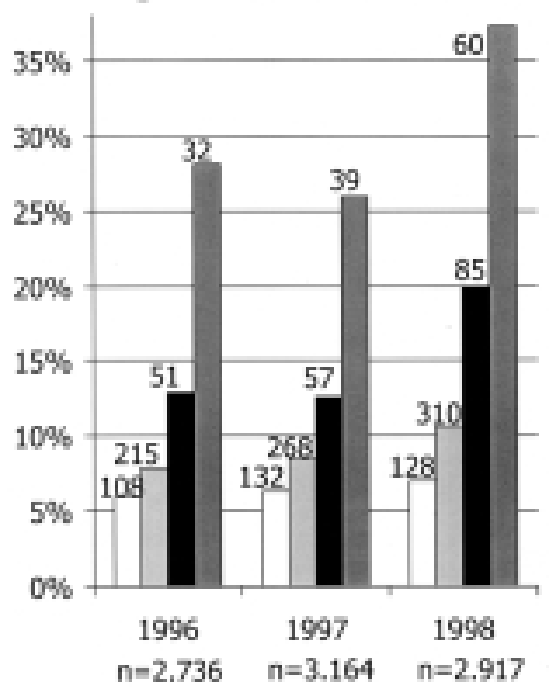

MDR
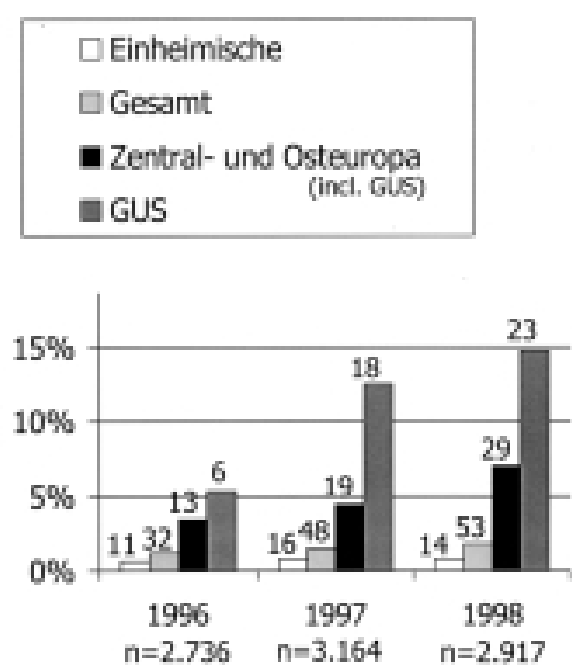

Abb. 13 Resistenzentwicklung von M. tuberculosis-Komplex für Erstrangmedikamente (DZK-Studie), Absolut und Prozent sind diese Ergebnisse nicht als repräsentativ für Gesamtdeutschland anzusehen.

Dieser Trend wird auch durch die Ergebnisse der DZK-Studie bestätigt $[6,7,8]$, allerdings auf niedrigerem Niveau. Die $\mathrm{Zu}$ nahme resistenter, und insbesondere multiresistenter, d.h. Resistenz gegen mindestens INH und RMP, Stämme, ist insbesondere auf die erhebliche Zunahme der Resistenzraten bei Patienten aus osteuropäischen Ländern zurückzuführen (Abb.13) [9]. Während die Resistenzsituation in der deutschen Bevölkerung vergleichsweise stabil war, ließ sich bei Patienten aus der GUS 1998 eine Zunahme der Multiresistenz auf 11,8\% verzeichnen (1996: 6,8\%, 1997: 11,8\%).

Die steigenden Resistenzraten deuten darauf hin, dass sich nun auch in Deutschland die Auswirkungen des weltweiten Anstiegs resistenter Tuberkulosestämme zeigen, wobei die Nähe zu Osteuropa hier eine besondere Rolle spielt. Diese Entwicklung verdeutlicht die Notwendigkeit, Patienten, die aus Ländern mit hohen Resistenzraten stammen und/oder bereits antituberkulotisch vorbehandelt wurden, bis zum
Erhalt des Resultates der Empfindlichkeitsprüfung streng zu isolieren und initial mit vier oder mit fünf Medikamenten unter Berücksichtigung der Vorgeschichte - zu behandeln [10] (Tab. 2).

\section{Zusammenfassung}

Die 1998 gemeldeten Tuberkulosefälle (alle Formen) zeigten im Vergleich zu 1997 einen Rückgang um 6,5\%. Der Rückgang an offener Tuberkulose (Absolutzahlen) betrug 4,6\%, an geschlossener Tuberkulose $10,4 \%$ und an extrapulmonaler Tuberkulose 5,9\%. Der Ausländeranteil betrug ähnlich wie im Vorjahr 31,5\%, bei den Kindertuberkulosen 58,6\%. Die Zahl der Tuberkuloseerkrankungen beim Kind stieg 1998 nach dem deutlichen Rückgang 1997 (-16,9\%) wieder um 2,1\% an. Das Erkrankungsrisiko ausländischer Kinder lag im Vergleich zu dem einheimischer Kinder um das 10fache höher (relatives Risiko: 10,5). Unverändert zeigte sich eine höhere Inzidenz im Alter, die ausländische Bevölkerung wies drei Altersgipfel auf.

Tab. 3 Resistenzen gegen Antituberkulotika (Umfrageergebnisse des AKM 1998)

\begin{tabular}{lllllllll}
\hline Jahr & $\begin{array}{l}\text { Zahl der teilnehmen- } \\
\text { den Labore }\end{array}$ & $\begin{array}{l}\text { Zahl der } \\
\text { Stämme }\end{array}$ & $\mathrm{H}_{\mathrm{r}} \%$ & $\mathrm{R}_{\mathrm{r}} \%$ & $\mathrm{~S}_{\mathrm{r}} \%$ & $\mathrm{E}_{\mathrm{r}} \%$ & $\mathrm{Z}_{\mathrm{r}}^{*} \%$ & $\mathrm{H}_{\mathrm{r}}+\mathrm{R}_{\mathrm{r}}\left(+\mathrm{X}_{\mathrm{r}}\right) \%$ \\
\hline 1991 & 32 & 2776 & 5,1 & 1,4 & 2,9 & 1,0 & - & 1,1 \\
1992 & 32 & 3345 & 5,6 & 1,5 & 5,0 & 1,0 & - & 1,0 \\
1993 & 25 & 2654 & 6,3 & $1,7 \mathrm{~s}$ & 5,0 & 1,9 & - & 1,4 \\
1994 & 27 & 2418 & 4,4 & 1,5 & 3,9 & 1,0 & - & 1,2 \\
1995 & 33 & 2579 & 5,5 & 1,6 & 4,7 & 2,0 & 1,4 & 1,3 \\
1996 & 40 & 3332 & 5,6 & 1,7 & 4,0 & 1,2 & 1,1 & 1,4 \\
1997 & 43 & 3267 & 7,1 & 2,5 & 4,7 & 2,3 & 1,7 & 2,1 \\
1998 & 28 & 2727 & 7,2 & 2,1 & 5,3 & 2,2 & 1,5 & 1,7 \\
\hline
\end{tabular}

$\mathrm{H}=\mathrm{INH}, \mathrm{R}=\mathrm{RMP}, \mathrm{S}=\mathrm{SM}, \mathrm{E}=\mathrm{EMB}, \mathrm{Z}=\mathrm{PZA}$

${ }_{*} M$. bovis wurde nicht gezählt 
Unter Berücksichtigung der im Rahmen der DZK-Studie „Epidemiologie der Tuberkulose“ erhobenen Daten, der Umfrageergebnisse des Arbeitskreises Mykobakterien sowie der vom Nationalen Referenzzentrum für Mykobakterien in Borstel registrierten Entwicklung muss auch in Deutschland von einer langsamen Zunahme der Resistenzen ausgegangen werden, wobei hier vor allem ausländische Bevölkerungsgruppen betroffen sind. Der Trend in der Zunahme (multi)resistenter $M$. tuberculosis-Stämme gegen Erstrangmedikamente setzte sich 1998 fort. Eine besondere Rolle spielte hierbei der Resistenzanstieg bei Bevölkerungsgruppen aus Zentral- und Osteuropa (insbesondere den GUS-Staaten). Unter Berücksichtigung der Verschlechterung der Resistenzlage ist auf die zwingende Therapieeinleitung mit einer 4- oder 5-Fachkombination bei Neuerkrankungen dringend hinzuweisen.

Entscheidend für die Kontrolle der Tuberkulosesituation im eigenen Land ist auch weiterhin die Erfassung und aufmerksame Beobachtung aktueller epidemiologischer Trends inklusive der Resistenzraten. Das allgemeine Erkrankungsrisiko ist zurückgegangen, während sich das Risiko für bekannte Risikogruppen erhöht hat. Daher sind neben der Identifikation gefährdeter Personengruppen und der Umgebungsuntersuchungen sowohl ein zielgruppenorientierter Diagnostik- und Therapieansatz als auch angepasste Präventionsmaßnahmen erforderlich. Das neue Infektionsschutzgesetz („Seuchenneuordnungsgesetz“) wird hier wertvolle Hilfe leisten.

Die Zunahme der (Multi)-Resistenzen in Osteuropa ist auch für die Bevölkerung Deutschlands von Bedeutung und erfordert eine stärkere Beteiligung an internationalen Tuberkulosekontrollprogrammen.

\section{Danksagung}

Die statistischen Daten wurden freundlicherweise vom Statistischen Bundesamt Wiesbaden und den statistischen Landesämtern zur Verfügung gestellt. Die DZK-Studienergebnisse zur Resistenz wurden von Herrn Dr. Forßbohm/Gesundheitsamt Wiesbaden in Zusammenarbeit mit 285 Gesundheitsämtern in Deutschland erhoben. Resistenzdaten wurden uns auch vom Arbeitskreis Mykobakterien sowie von Frau Dr. Rüsch-Gerdes vom Nationalen Referenzzentrum in Borstel zur Verfügung gestellt. Wir bedanken uns ganz herzlich für das Engagement und den Arbeitseinsatz aller Beteiligten. Unser besonderer Dank gilt dem Bundesministerium für Gesundheit für die Unterstützung des DZK.

\section{Literatur}

${ }^{1}$ Deutsches Zentralkomitee zur Bekämpfung der Tuberkulose. 25. Informationsbericht. Frankfurt/Main: Pmi Verlagsgruppe (erhältlich beim DZK), 1999

${ }^{2}$ Statistisches Bundesamt Wiesbaden. - VII B-176

${ }^{3}$ Statistisches Jahrbuch 1999 für die Bundesrepublik Deutschland. Stuttgart: Metzler-Poeschel Verlag

${ }^{4}$ Ständige Impfkommission am Robert-Koch-Institut (STIKO). Impfempfehlungen. Epidemiologisches Bulletin des RobertKoch-Instituts, 1998; 15: 109-112

${ }^{5}$ Auswertung von R. Küchler (Berlin), U. Müller-Brundaler und R. Urbanczik (Schiffweiler); Korrespondenz bitte an Dr. R. Küchler, Krankenhaus Neukölln, Rudower Str. 98, 12351 Berlin
${ }^{6}$ Forßbohm M. Studie des DZK zur Epidemiologie der Tuberkulose - Zwischenbericht 1996. In: Deutsches Zentralkomitee zur Bekämpfung der Tuberkulose, 23. Informationsbericht. Frankfurt/Main: Pmi Verlagsgruppe (erhältlich beim DZK), 1997

${ }^{7}$ Forßbohm M. Studie des DZK zur Epidemiologie der Tuberkulose - Zwischenbericht 1997. In: Deutsches Zentralkomitee zur Bekämpfung der Tuberkulose, 24. Informationsbericht. Frankfurt/Main: Pmi Verlagsgruppe, 1998

${ }^{8}$ Robert-Koch-Institut. Zur Struktur der Tuberkulosemorbidität in Deutschland - Ergebnisse der Studie des DZK zur Epidemiologie der Tuberkulose. Epidemiologisches Bulletin 1998; 49: 349-351

${ }^{9}$ Loddenkemper R, Hauer B, Sagebiel D, Forßbohm M. Tuberkuloseepidemiologie in Deutschland und der Welt mit Schwerpunkt Osteuropa. Bundesgesundheitsblatt 1999; 42: 683-693

${ }^{10}$ Iseman MD. Treatment of multidrug-resistant tuberculosis. N Engl J Med 1993; 329: 784 - 791

${ }^{11}$ Magdorf K. Gezielte Tuberkulintestung und Chemoprävention bei Kindern. Die wichtigste Maßnahme zur Tuberkulose-Prävention, Tb-Letter. 10 1999: 1

12 Brodehl J. Kommission für Infektionskrankheiten und Impffragen in Zusammenarbeit mit der DGPI; Tuberkulintestung im Kindesalter. Monatsschrift für Kinderheilkunde (MoKi). 7/1999: $691-692$

\section{Generalsekretär Prof. Dr. R. Loddenkemper}

Deutsches Zentralkomitee zur Bekämpfung der Tuberkulose Lungenklinik Heckeshorn

Zum Heckeshorn 33

14109 Berlin

E-mail: loddheck@zedat.fu-berlin.de 